In the simplest case, when the peaks are well resolved without overlapping in an orthogonal cell of a centrosymmetric structure, the atomic coordinate errors are obtained by dividing the slope error by the peak curvatures. For non-centrosymmetric structures the divisor is a function such as $(3 \cdot 16)$ of Cruickshank (1952). An exact discussion of coordinate errors under general conditions has been given by Cruickshank \& Robertson (1953).

\section{Conditions of validity}

For positions close to but not exactly on special positions the density and slope errors are intermediate between the special and general values, and it may be necessary to use the exact formulae (3) and (8). 'Close' here means within approximately half the distance apart at which two point atoms would be resolved in a similar Fourier analysis containing terms of the same indices.
The approximate formulae also depend on there being a large number of terms in the series, and on there being no plane with $\sigma(F)$ much greater than for any others. The approximate formulae should ordinarily be sufficient if there are more than about 30 different sub-forms.

One of us (J.S.R.) wishes to acknowledge a Further Education and Training Grant from the Ministry of Education, which enabled him to take part in this work.

\section{References}

Cruickshank, D. W. J. (1949a). Acta Cryst. 2, 65. Cruickshank, D. W. J. (1949b). Acta Cryst. 2, 154.

Cruickshank, D. W. J. (1952). Acta Cryst. 5, 511.

Cruickshank, D. W. J. \& Robertson, A.P. (1953). Acta Cryst. 6, 698.

Jeffrey, G. A. \& Rollett, J. S. (1952). Proc. Roy. Soc. A, 213, 86 .

Acta Cryst. (1953). 6, 707

\title{
The Crystal Structure of $\alpha$-Thiopyridone
}

\author{
By Bruce R. Penfold* \\ Crystallographic Laboratory, Cavendish Laboratory, Cambridge, England
}

(Received 4 March 1953)

\begin{abstract}
The crystal structure of $\alpha$-thiopyridone has been determined by means of electron-density projections on the (001) and $(010)$ planes. The $(001)$ projection was solved by direct determination of the signs of Fourier coefficients. Bond lengths of the pyridine ring are interpreted in terms of a resonance structure and hence a value is deduced for the bond order of the $\mathrm{C}-\mathrm{S}$ bond. The $\mathrm{C}-\mathrm{S}$ order/length curve is discussed with reference to the results from this structure analysis and that of thiophthen. $\alpha$-Thiopyridone molecules are linked in pairs across centres of symmetry by what appear to be weak hydrogen bonds between nitrogen and sulphur atoms. There are several points of similarity between this structure and that of $\alpha$-pyridone.
\end{abstract}

\section{Introduction}

In a previous communication (Penfold, 1953), the crystal structure of the tautomeric compound $\alpha$ pyridone was reported, the existence of the pyridone tautomer being established by accurate electrondensity measurements which led to direct location of hydrogen atoms. The molecule of $\alpha$-thiopyridone, which differs from that of $\alpha$-pyridone only in that the oxygen atom has been replaced by a sulphur atom, is also tautomeric in a similar way, the two forms being shown in Fig. 1, I being thiopyridone and II thiolpyridine. It is therefore of some interest to compare its crystal structure with that of $\alpha$-pyridone. The possibility of a reliable determination of the C-S bond length is of some importance because of the addition

* Now at Canterbury University College (University of New Zealand), Christchurch C.1, New Zealand. it would provide to the very meagre body of informa-<smiles>S=c1cccc[nH]1</smiles><smiles>Sc1cccnc1</smiles>

Fig. 1. Tautomeric forms of the molecule. 
tion concerning the order/length relationship in such bonds.

\section{Experimental}

The material used was kindly supplied by Prof. L. Hunter of University College, Leicester, in the form of very small yellow needles. Recrystallization from benzene yielded larger crystals in two different habits, needles elongated parallel to the $b$ axis, and thin plates with greatest elongation parallel to the $c$ axis and greatest development of the (100) face.

\section{Crystallographic and physical data} $\alpha$-Thiopyridone, $\mathrm{C}_{5} \mathrm{NSH}_{5}$; monoclinic,

$$
\begin{gathered}
a=14 \cdot 35, b=6 \cdot 31, c=6 \cdot 10 \AA \text { (all } \pm 0 \cdot 3 \% \text { ) } \\
\beta=103^{\circ} 01^{\prime} \pm 5^{\prime} .
\end{gathered}
$$

Absent spectra: $h 0 l$ and $0 k 0$ when $h$ and $k$, respectively, are odd. Space group $P 2_{1} / a$. Four molecules per unit cell. Density (calc.) $=1.375$ g.cm. ${ }^{-3}$. Absorption coefficient for $\mathrm{Cu} K \alpha$ radiation, $\mu=42 \mathrm{~cm}^{-1}$.

Complete sets of relative intensities of $h 0 l$ and $h k 0$ spectra were obtained using normal Weissenberg techniques with visual estimation of intensities. To the $h k 0$ set of intensities only, for which a plateshaped crystal was used, corrections were made for spot shape as suggested by Broomhead (1948). No absorption corrections were applied. All intensities were converted to an absolute scale by the statistical method of Wilson (1942), and values of the unitary structure factors, $|U(h k l)|$, were calculated (Kasper, Lucht \& Harker, 1950).

\section{Patterson projections}

Unsuccessful attempts were made to determine the coordinates of the sulphur atom from sharpened Patterson projections on (001) and (010). The reasons for failure, which appeared later, were first that the S-S peak in a general position in the (001) projection was no higher than several other chance peaks, and secondly that in the $(010)$ projection the S-S peak occurred adjacent to the peak of equal height corresponding to the vector between centrosymmetrically related pyridine rings. Without a knowledge of the sulphur position, trial-and-error methods using structure factor graphs are impracticable because the values of structure factors are so very sensitive to the position of the heary atom.

\section{Determination of the signs of the coefficients $\boldsymbol{F}(\boldsymbol{h k} \mathbf{0})$}

It has been shown (Cochran, 1952) that the relation

$$
S(h k l)=S\left(h^{\prime} k^{\prime} l^{\prime}\right) S\left(h+h^{\prime}, k+k^{\prime}, l+l^{\prime}\right),
$$

where $S(h k l)$ denotes the sign of $F(h k l)$, is very probably true when $\sqrt{ }(3 n) . \bar{U}>1, n$ being the symmetry num- ber and $\bar{U}$ the r.m.s. value of the three unitary structure factors $U(h k l), U\left(h^{\prime} k^{\prime} l^{\prime}\right)$ and $U\left(h+h^{\prime}, k+k^{\prime}, l+l^{\prime}\right)$. It was used on the $h k 0$ coefficients of this structure in much the same way as in the successful solution of one projection of the crystal structure of L-glutamine (Cochran \& Penfold, 1952). The presence of the comparatively heavy sulphur atom is an advantage as it makes the average value of $|U|$ for all spectra fairly high, and some individual values particularly high, circumstances favourable for the use of equation (1). The value of $\overline{U^{2}}$ is equal to $\left\{\sum_{j=1}^{N} n_{j}^{2}\right\}^{1 / 2}$, where $n_{j}$ is the fraction of electrons in the cell associated with the $j$ th atom, and for this structure has the value $0 \cdot 21$ compared with $0 \cdot 16$ for glutamine. There are nineteen $|U(h k 0)|$ values greater than 0.30 and of these, six are greater than 0.40 and three are greater than 0.50. Clearly Harker-Kasper inequalities (Harker \& Kasper, 1948) can be expected to establish some signs or sign relationships, but the inequalities were not tested at the initial stage.

$|U(25)|=0.51$ and $|U(34)|=0.32$ were first assumed to be positive, thus fixing the origin. Then, use was made of the fact that for this space group

$$
\begin{aligned}
& S(h k)=S(\bar{h} \bar{k})=-S(h \bar{k})=\frac{-S(\bar{h} k)}{} \\
& \text { when }(h+k) \text { is odd } \\
& \text { and } \\
& S(h k)=S(\bar{h} \bar{k})=S(h \bar{k})=\begin{array}{l}
S(\bar{h} k) \\
\text { when }(h+k) \text { is even . }
\end{array}
\end{aligned}
$$

Equations (2) and (3) were used in the following way:

$$
S(06)=S(14,3) S(\overline{14}, 3)
$$

where $|U(06)|=0 \cdot 78$ and $|U(14,3)|=0 \cdot 55$. Therefore $S(06)$ is negative, a result confirmed by high values for $|U(83)|$ and $|U(10,3)|$. Similarly $S(40)$ was shown to be negative. Thence, by assuming $S(60)=a$ and

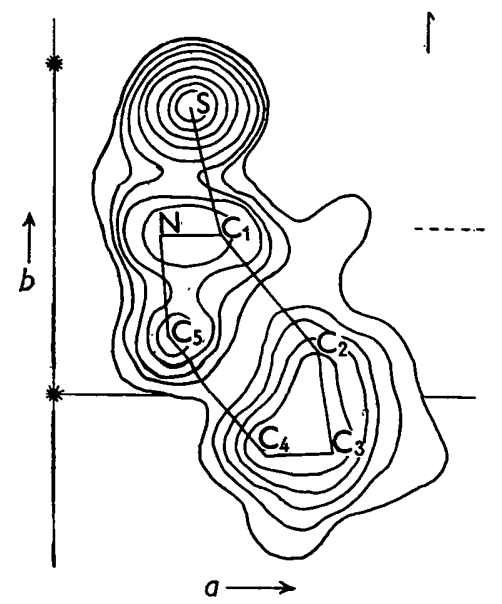

Fig. 2. First electron-density projection on (001) with the final position of the molecule indicated. In this synthesis only those 41 terms were included whose signs had been determined by sign relationships. Arbitrary contour interval doubled about sulphur atom. 
$S(14,3)=b$, sign determination proceeded as a chain process by use of equation (l) until probable signs had been given to 41 structure factors (including all for which $|U|$ was $>0 \cdot 30$ ) out of a total of 117 . Most were in terms of $a$ and/or $b$ but there were strong indications that $a$ was negative. Therefore, using these 41 terms, two Fourier syntheses were evaluated, one with $a=-$ and $b=-$, the other with $a=-$ and $b=+$. The first sign combination gave a map which could not be interpreted in terms of the $\alpha$-thiopyridone molecule, but the map corresponding to the second combination (Fig. 2) showed a clear picture of the molecule, and the large majority of the signs were clearly correct.

Approximate $x$ and $y$ atomic coordinates were assigned from this map. After subsequent refinement, it was shown that only one sign of the original 41 , that of $|U(35)|=0 \cdot 14$, had been incorrectly assigned.

An investigation was made of the amount of progress which could have been made at the initial stage by the use of Harker-Kasper inequalities. It appeared that they would have determined absolutely only two signs, $S(40)$ and $S(06)$, and would have given in addition four simple sign relationships involving seven different structure factors. Thus the sign-determining process could have been initiated but could not have been carried far enough to determine the structure for certain.

\section{Electron-density projections}

Refinement of the (001) projection proceeded by the use of successive $\left(F_{o}-F_{c}\right)$-syntheses. The sulphur atom was assigned a greater-than-average temperature factor of $\exp \left[-0.5 \sin ^{2} \theta\right]$, but all other atoms were given the average molecular value of $\exp \left[-1 \cdot 3 \sin ^{2} \theta\right]$.

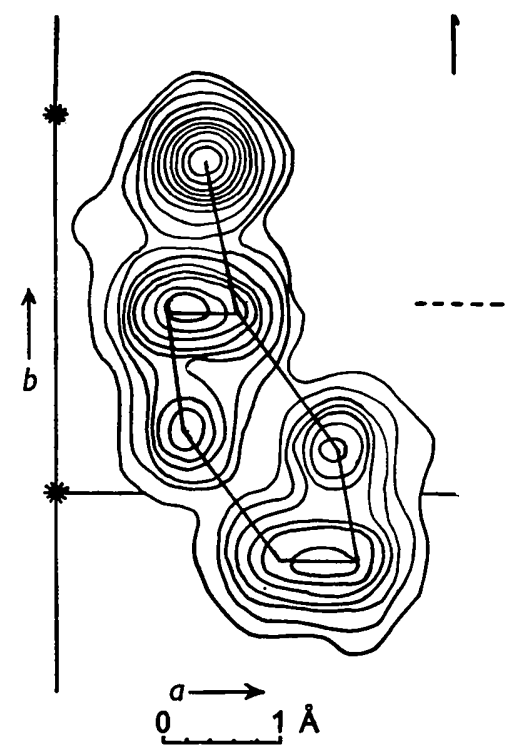

Fig. 3. Final electron-density projection of one molecule on (001). Contours at an interval of $1 \cdot 0$ e. $\AA^{-2}\left(2 \cdot 0\right.$ e. $\AA^{-2}$ about $\left.S\right)$, starting at $2 \cdot 0 \Theta . \AA^{-2}$.
Hydrogen atoms were allowed for in $F$ calculations by placing them at calculated covalent bond distances from the appropriate ring atoms. The factor $R=$ $\Sigma\left|F_{o}-F_{c}\right| \div \Sigma\left|F_{o}\right|$ was reduced to $0 \cdot 136$ or, if zero $F_{o}$ values are omitted, $0 \cdot 124$. The final electron-density projection on (001) is shown in Fig. 3.

Relative $z$ coordinates were assigned by clamping a model of the planar molecule in the correct position over the known (001) projection. The correct translation of the molecule was fixed because, with $x$ and $y$ coordinates fixed, the S-S peaks on the two Patterson projections could be determined and hence the $z$ coordinate of the sulphur atom. $h 0 l$ structure factors were calculated and refinement of the $(010)$ projection proceeded as before. In this case, however, the thermal vibration of the sulphur atom is markedly anisotropic, as can be observed from the appearance in one region of the first $D$ map (Fig. 4), and the final greater-thanaverage temperature factor assigned to it was $\exp \left[-1.2 \sin ^{2}\left(\varphi-45^{\circ}\right) \sin ^{2} \theta\right]$ (Hughes, 1941), indi-

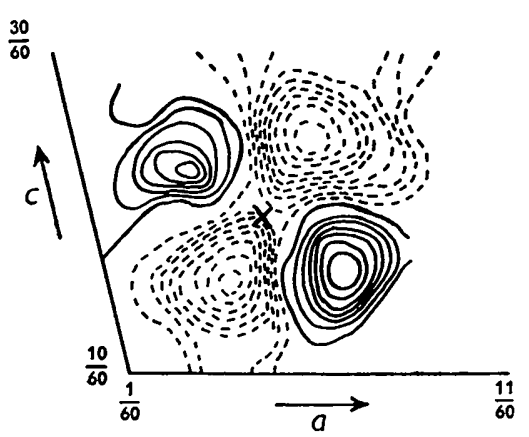

Fig. 4. The first $D$ map projected on $(010)$ in the region of the sulphur atom (position marked with cross). Contours at an interval of 0.2 e. $\AA^{-2}$, starting at 0.2 e. $\AA^{-2}$; negative contours broken.

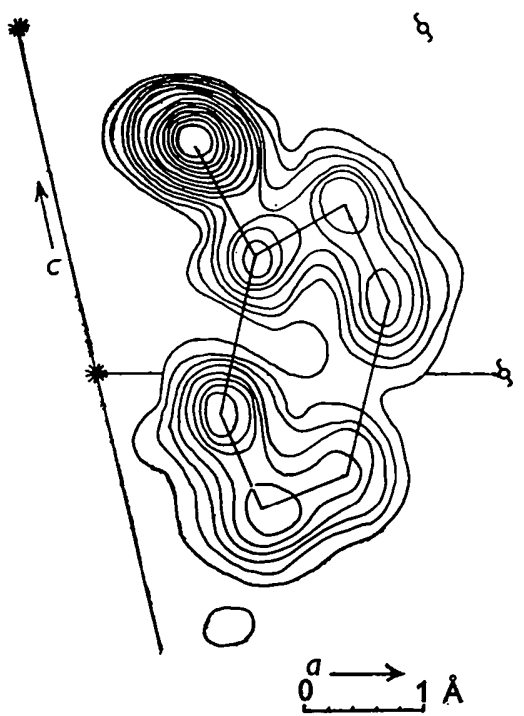

Fig. 5. Final electron-density projection of one molecule on (010). Contours at an interval of 1.0 e. $\AA^{-2}\left(2 \cdot 0\right.$ e. $\AA^{-2}$ about $\left.S\right)$, starting at $2 \cdot 0$ e. $\AA^{-2}$. 
cating that the maximum vibration direction makes an angle of $45^{\circ}$ with the $c$ axis. The appearance of final $D$ maps in regions remote from atoms was rather better than for the (001) projection and it was felt justified to apply greater-than-average temperature factors to $\mathrm{C}_{3}$ of $\exp \left[-0 \cdot 6\left(\frac{1}{2} l c^{*}\right)^{2}\right]$ and to $\mathrm{C}_{4}$ of $\exp \left[-0 \cdot 3 \sin ^{2} \theta\right]$. The average molecular temperature factor was $\exp \left[-1 \cdot 1 \sin ^{2} \theta\right]$. The factor $R$ was reduced to 0.116 or, if zero $F_{o}$ values are omitted, $0 \cdot 100$. The final electron-density projection on (010) is shown in Fig. 5. Coordinates of atoms other than hydrogen are listed in Table 1 , and observed and calculated structure factors for the $h k 0$ and $h 0 l$ zones are compared in Tables 3 and 4.

Table 1. Atomic coordinates

$\begin{array}{clcr}\text { Atom } & x(\AA) & y(\AA) & z(\AA) \\ \mathrm{S} & 1.327 & 2.756 & 2 \cdot 026 \\ \mathrm{~N} & 1.00 & 1.51 & -0.36 \\ \mathrm{C}_{1} & 1.64 & 1.50 & 1.03 \\ \mathrm{C}_{2} & 2.53 & 0.37 & 1.46 \\ \mathrm{C}_{3} & 2.69 & -0.58 & 0.63 \\ \mathrm{C}_{4} & 1.98 & -0.57 & -0.88 \\ \mathrm{C}_{5} & 1.16 & 0.53 & -1.20\end{array}$

The standard deviation (s.d.) in electron density, $\sigma(\varrho)$, was determined as by Cochran (1948) and the s.d. in coordinate, $\sigma\left(x_{i}\right)$, as by Cox \& Cruickshank (1948). Results were:

$$
\sigma(\varrho)=0 \cdot 30 \text { e. } \AA^{-2} \pm 10 \% \text { for both projections. }
$$

The mean value of $\sigma\left(x_{i}\right)$ was the same for both projections, i.e.

$$
\begin{aligned}
& \sigma\left(x_{i}\right)=0.018 \AA \text { for } \mathrm{C} \text { and } \mathrm{N} \text { atoms, } \\
& \sigma\left(x_{i}\right)=0.006 \AA \text { for the } \mathrm{S} \text { atom. }
\end{aligned}
$$

S.d's in bond lengths, $\sigma(r)$, were hence as follows:

$$
\begin{aligned}
& \text { for } \mathrm{C}-\mathrm{C} \text { and } \mathrm{C}-\mathrm{N} \text { bonds, } \\
& \sigma(r)=V 2 \times 0.018=0.025 \AA ; \\
& \text { for the } \mathrm{C}-\mathrm{S} \text { bond, } \\
& \sigma(r)=\left(0.018^{2}+0.006^{2}\right)^{\frac{1}{2}}=0.020 \AA .
\end{aligned}
$$

We may therefore take the limit of error in these bond lengths to be $0.065 \AA$ and $0.050 \AA$ respectively, with a limit of error in bond angle of about $5^{\circ}$.

\section{Description and discussion of structure}

In Fig. 6 are shown the bond lengths and bond angles observed in this analysis. There are obvious errors in the lengths of $\mathrm{C}_{2}-\mathrm{C}_{3}$ and $\mathrm{C}_{3}-\mathrm{C}_{4}$, the former being observed as $0.04 \AA$ less than the theoretical pure double-bond length, and the latter as being virtually a pure single bond.

Although there is no direct evidence of the existence of the thiopyridone tautomer ( $\mathrm{I}$ in Fig. 1) rather than the thiolpyridine, the former is much more likely both by analogy with $\alpha$-pyridone and by consideration of the length of the $\mathrm{C}_{1}-\mathrm{S}$ bond. This bond is observed as $0.13 \AA$ shorter than the sum of the Pauling covalent radii for carbon and sulphur, and therefore must

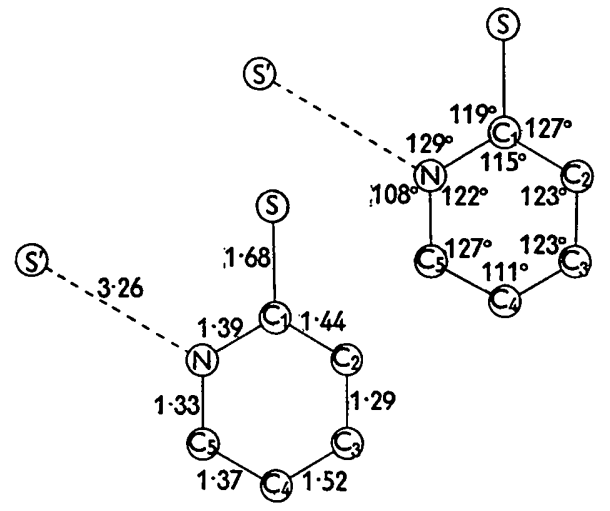

Fig. 6. Bond lengths (in Ångström units) and bond angles. $\mathrm{S}^{\prime}$ is the atom to which $\mathbf{N}$ has been assumed to be hydrogen bonded.

possess considerable double character, a fact which makes the existence of tautomer II most unlikely.

\section{Significance of the carbon-sulphur bond}

The relationship between order and length for C-S bonds has been discussed by Cox, Gillot \& Jeffrey (1949), who have also summarized experimental evidence for the lengths of the pure single and double bonds, and their paper will be referred to in the ensuing discussion. The order/length curve for C-S bonds is not known with sufficient accuracy for us to deduce the bond order of the $\mathrm{C}_{1}-\mathrm{S}$ bond in $\alpha$-thiopyridone directly from its length. We may, however, consider the remaining bond lengths and, assuming tautomer I, calculate the weights of the different possible resonance contributors which lead to best agreement with observed bond lengths. An indication of the bond order for $\mathrm{C}_{1}-\mathrm{S}$ is then obtained. The possible resonance contributors are analogous to those listed for $\alpha$-pyridone (Penfold, 1953) and it was found that the best weights are exactly those of set $(b)$ chosen for this compound. Bond lengths, $r_{c}$, calculated for this set are compared with those observed, $r_{o}$, in both $\alpha$-pyridone and $\alpha$-thiopyridone in Table 2. For thiopyridone, the r.m.s. value of $\left|r_{o}-r_{c}\right|$ is $0.04 \AA$,

Table 2. Observed and calculated bond lengths in the pyridine rings of $\alpha$-thiopyridone and $\alpha$-pyridone

$\begin{array}{lccc} & & \alpha \text {-Thiopyridone } & \alpha \text {-Pyridone } \\ \text { Bond } & r_{c}(\AA) & r_{o}(\AA) & r_{o}(\AA) \\ \mathrm{C}_{1}-\mathrm{C}_{2} & 1.45 & 1.44 & 1 \cdot 44 \\ \mathrm{C}_{2}-\mathrm{C}_{3} & 1.35 & 1 \cdot 29 & 1 \cdot 33 \\ \mathrm{C}_{3}-\mathrm{C}_{4} & 1 \cdot 44 & 1.52 & 1 \cdot 42 \\ \mathrm{C}_{4}-\mathrm{C}_{5} & 1.37 & 1.37 & 1 \cdot 37 \\ \mathrm{C}_{5}-\mathrm{N} & 1.33 & 1 \cdot 33 & 1 \cdot 34 \\ \mathrm{~N}-\mathrm{C}_{1} & 1.40 & 1.39 & 1 \cdot 40\end{array}$

$r_{c}$ values are those calculated for set $(b)$ of the weights of resonance contributors listed for the structure of $\alpha$-pyridone (Penfold, 1953). 
Table 3. Observed and calculated structure factors for the $h k 0$ zone

$F_{c}$ values were derived using the $f$ values listed by James \& Brindley (1931) to which were applied the average molecular temperature factor and the greater-than-average atomic temperature factors

\begin{tabular}{|c|c|c|c|c|c|c|c|c|c|c|c|}
\hline$h$ & $k$ & $F_{o}$ & $F_{c}$ & $h$ & $k$ & $F_{o}$ & $F_{c}$ & $h$ & $k$ & $F_{o}$ & $F_{c}$ \\
\hline 0 & 0 & - & 232 & 14 & & $3 \cdot 6$ & $-3 \cdot 3$ & 3 & & $6 \cdot 8$ & $6 \cdot 7$ \\
\hline 2 & & $17 \cdot 4$ & $20 \cdot 2$ & 15 & & 1.6 & 1.7 & 4 & & $14 \cdot 0$ & $-14 \cdot 6$ \\
\hline 4 & & $61 \cdot 6$ & -66.0 & 16 & & $6 \cdot 0$ & $-4 \cdot 2$ & 5 & & 7.6 & $-7 \cdot 2$ \\
\hline 6 & & $35 \cdot 2$ & -35.5 & 17 & & 0 & $-0 \cdot 1$ & 6 & & $4 \cdot 4$ & $3 \cdot 5$ \\
\hline 8 & & $17 \cdot 8$ & $-16 \cdot 2$ & & & & & 7 & & $4 \cdot 4$ & $-3 \cdot 1$ \\
\hline 10 & & $20 \cdot 6$ & 19.8 & 1 & 3 & $12 \cdot 0$ & $-12 \cdot 3$ & 8 & & 12.8 & 12.6 \\
\hline 12 & & $14 \cdot 8$ & $15 \cdot 3$ & 2 & & 8.8 & -10.0 & 9 & & $2 \cdot 4$ & $4 \cdot 2$ \\
\hline 14 & & $3 \cdot 6$ & $-2 \cdot 2$ & 3 & & $2 \cdot 4$ & & 10 & & $7 \cdot 2$ & $7 \cdot 8$ \\
\hline 16 & & 0 & 0 & 4 & & $12 \cdot 8$ & -15.2 & 11 & & 0 & 0.9 \\
\hline & & & & 5 & & 12.4 & 13.4 & 12 & & $4 \cdot 8$ & -4.5 \\
\hline 1 & 1 & $3 \cdot 2$ & 0 & 6 & & 0 & -0.3 & 13 & & $2 \cdot 0$ & -0.4 \\
\hline 2 & & $43 \cdot 2$ & $-47 \cdot 4$ & 7 & & 4.4 & $5 \cdot 2$ & 14 & & $3 \cdot 6$ & -3.5 \\
\hline 3 & & $33 \cdot 2$ & $-29 \cdot 6$ & 8 & & $14 \cdot 4$ & $16 \cdot 1$ & & & & \\
\hline 4 & & $42 \cdot 4$ & $-47 \cdot 4$ & 9 & & 4.8 & -4.9 & 0 & 6 & $22 \cdot 4$ & $-26 \cdot 2$ \\
\hline 5 & & $35 \cdot 2$ & $38 \cdot 0$ & 10 & & $10 \cdot 0$ & $11 \cdot 2$ & 1 & & 0 & $-1 \cdot 6$ \\
\hline 6 & & 2.0 & 2.7 & 11 & & $3 \cdot 2$ & -2.9 & 2 & & $3 \cdot 6$ & -3.9 \\
\hline 7 & & $11 \cdot 6$ & 11.4 & 12 & & $5 \cdot 6$ & -77 & 3 & & 4.0 & 4.8 \\
\hline 8 & & $19 \cdot 6$ & $19 \cdot 8$ & 13 & & 0 & -0.7 & 4 & & $11 \cdot 6$ & $12 \cdot 8$ \\
\hline 9 & & 18.0 & $-17 \cdot 3$ & 14 & & $11 \cdot 6$ & $-12: 0$ & 5 & & $2 \cdot 0$ & $3 \cdot 6$ \\
\hline 10 & & $9 \cdot 6$ & $9 \cdot 2$ & 15 & & 0 & 0.5 & 6 & & $7 \cdot 6$ & $7 \cdot 4$ \\
\hline 11 & & $7 \cdot 6$ & -7.3 & 16 & & 0 & 0.6 & 7 & & $6 \cdot 4$ & -5.7 \\
\hline 12 & & $2 \cdot 8$ & $-2 \cdot 9$ & & & & & 8 & & $4 \cdot 4$ & 3.5 \\
\hline 13 & & $1 \cdot 6$ & $-2 \cdot 4$ & 0 & 4 & $2 \cdot 0$ & $3 \cdot 5$ & 9 & & $2 \cdot 4$ & $-2 \cdot 0$ \\
\hline 14 & & 3.2 & $-4 \cdot 1$ & 1 & & $12 \cdot 4$ & $12 \cdot 3$ & 10 & & 2.0 & $-4 \cdot 4$ \\
\hline 15 & & $6 \cdot 4$ & 7.9 & 2 & & 6.0 & 4.9 & 11 & & $2 \cdot 4$ & 0.9 \\
\hline 16 & & 0 & 1.1 & 3 & & $21 \cdot 6$ & 21.8 & 12 & & $3 \cdot 2$ & -3.7 \\
\hline 17 & & $5 \cdot 6$ & $4 \cdot 0$ & 4 & & $.3 \cdot 2$ & $-2 \cdot 2$ & & & & \\
\hline & & & & 5 & & 0 & -0.5 & 1 & 7 & 0 & $-1 \cdot 1$ \\
\hline 0 & 2 & $28 \cdot 4$ & $23 \cdot 8$ & 6 & & $8 \cdot 0$ & -7.7 & 2 & & 0 & -1.2 \\
\hline 1 & & $22 \cdot 0$ & $21 \cdot 0$ & 7 & & $14 \cdot 2$ & -14.9 & 3 & & $2 \cdot 8$ & 3.7 \\
\hline 2 & & $8 \cdot 4$ & $\begin{array}{r}-6.9 \\
\end{array}$ & 8 & & $\begin{array}{r}3 \cdot 6 \\
\end{array}$ & 0.2 & 4 & & 4.0 & $5 \cdot 6$ \\
\hline$\overline{3}$ & & 16.0 & $16 \cdot 0$ & 9 & & $7 \cdot 2$ & $-5 \cdot 4$ & 5 & & $5 \cdot 2$ & $-4 \cdot 1$ \\
\hline 4 & & $12 \cdot 4$ & -11.8 & 10 & & 0 & $\begin{array}{l}0.6 \\
0\end{array}$ & 6 & & 1.2 & \\
\hline 5 & & $8 \cdot 4$ & $-7 \cdot 1$ & 11 & & 4.0 & $4 \cdot 3$ & 7 & & $2 \cdot 0$ & $-\mathrm{I} \cdot 4$ \\
\hline 6 & & $2 \cdot 4$ & $-0 \cdot 3$ & 12 & & $\begin{array}{l}1.6 \\
1.6\end{array}$ & -0.3 & 8 & & 1.2 & $-2 \cdot 3$ \\
\hline 7 & & 10.0 & $-10 \cdot 2$ & 13 & & $2 \cdot 0$ & & 9 & & $2 \cdot 0$ & $3 \cdot 5$ \\
\hline 8 & & $3 \cdot 6$ & -4.9 & 14 & & 0 & $-1 \cdot 1$ & & & & \\
\hline $\begin{array}{l}\circ \\
9\end{array}$ & & 3.2 & $\begin{array}{l}-3.5 \\
-3.5\end{array}$ & $\begin{array}{l}15 \\
15\end{array}$ & & 2.0 & $\begin{array}{l}1.9 \\
\end{array}$ & 0 & 8 & $3 \cdot 6$ & -4.2 \\
\hline 10 & & $10 \cdot 0$ & $10 \cdot 1$ & & & & & 1 & & 0 & -1.2 \\
\hline 11 & & $4 \cdot 0$ & 5.6 & 1 & 5 & 0 & 0 & 2 & & 0 & 0.3 \\
\hline 12 & & 8.8 & 8.9 & 2 & & 22.8 & $-25 \cdot 6$ & 3 & & $1 \cdot 6$ & $2 \cdot 8$ \\
\hline 13 & & $\begin{array}{l}0.0 \\
3 \cdot 2\end{array}$ & $\begin{array}{l}2.6 \\
2 \cdot 6\end{array}$ & & & & & & & & \\
\hline
\end{tabular}

which is rather higher than the s.d. in the observed bond lengths, but the discrepancy is due entirely to the two bonds $\mathrm{C}_{2}-\mathrm{C}_{3}$ and $\mathrm{C}_{3}-\mathrm{C}_{4}$, the mean value of $\left|r_{o}-r_{c}\right|$ being only $0.027 \AA$. This is a rather unsatisfactory state of affairs, but it is felt that the excellent agreement for the other four observed bond lengths of the ring, both with calculated values and with those observed in $\alpha$-pyridone, is a strong indication that the weights assigned represent well the resonance contributions to this structure. Such a combination of weights indicates a double-bond character for $\mathrm{C}_{1}-\mathrm{S}$ of $65 \pm 5 \%$.

We are now able to define a point on the C-S order/length curve between those which correspond to pure single and pure double bonds. As can be seen from Fig. 7, the point we have obtained lies, within experimental error, on the lower straight line joining the two extreme points corresponding to pure single and pure double bonds which were arrived at by Cox et al. This straight line, however, does not fit the points which were obtained by comparison of the bond orders calculated for thiophthen by Evans \& de Heer (1949) with those observed by Cox et al. It seems unlikely, by analogy with other bond-order/length/energy curves (e.g. Skinner, 1945) that the true curve would

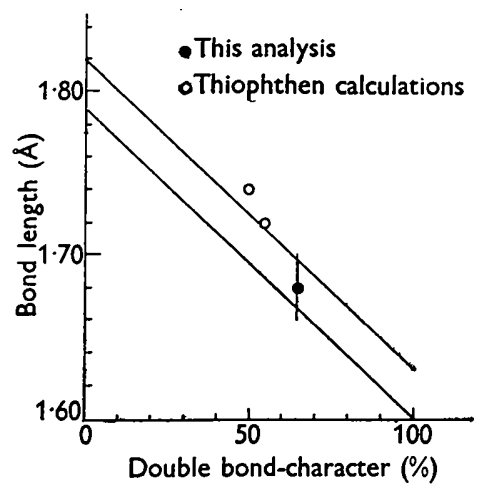

Fig. 7. C-S bond length as a function of bond order. 
Table 4. Observed and calculated structure factors for the hol zone

$F$ values here refer only to two molecules instead of four as in the $h k 0$ figures, the unit cell being halved in projection on (010). $h$ indices listed refer to this halved cell.

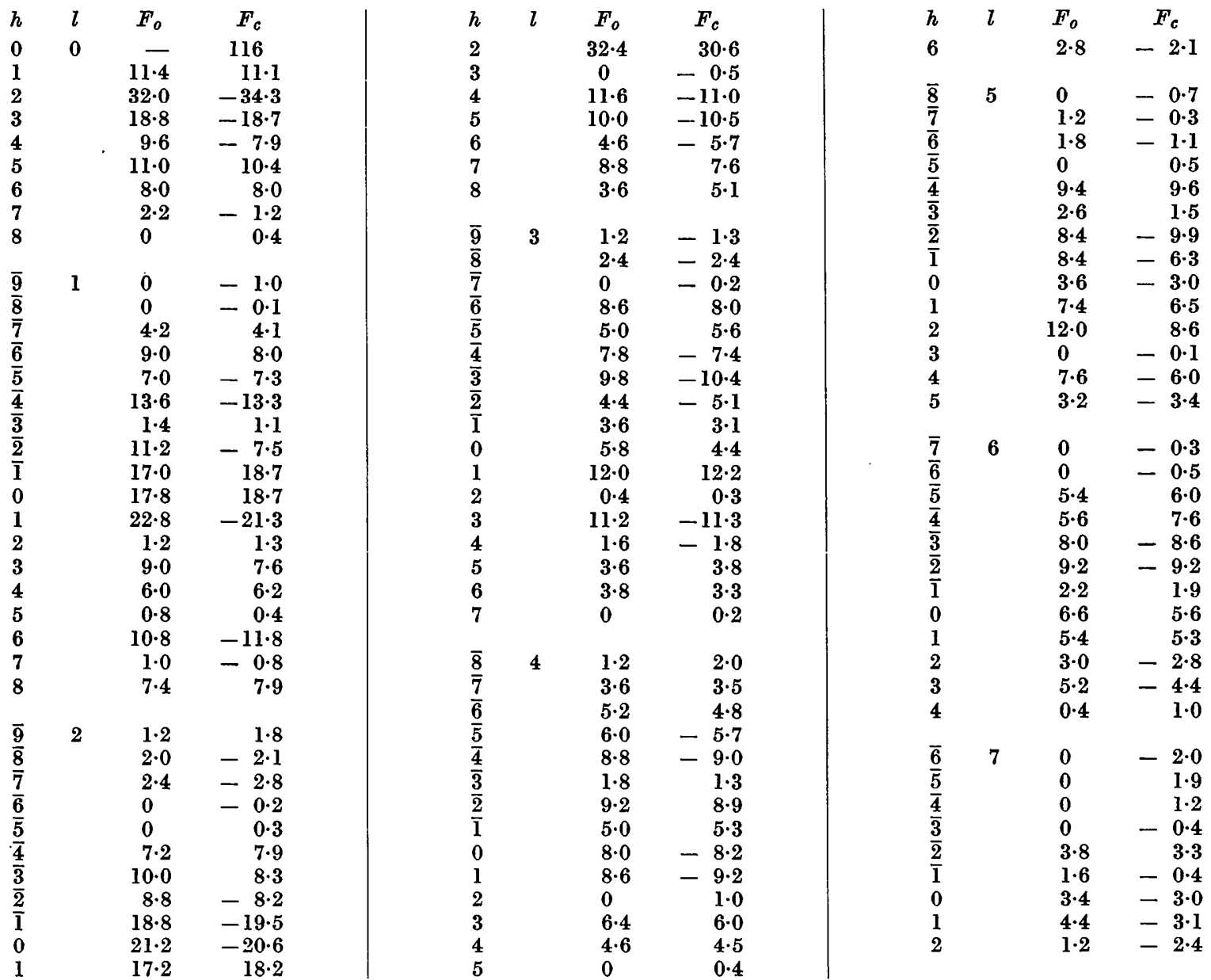

be concave to the axes, and Cox et al. have suggested that the single- and double-bond lengths may be greater than those they originally assumed. Thus an increase of $0.03 \AA$ in each of these assumed lengths would lead to a fit for the thiophthen points, assuming a straight-line plot. This alternative plot is shown in Fig. 7 (upper line) and it can be seen that it fits the thiopyridone point almost as well as the first plot. Clearly it is desirable to have a rather more accurate estimate of all the thiopyridone bond lengths so that an intermediate point on the C-S order/length curve can be fixed with some precision.

\section{Molecular environment}

Figs. 8 and 9 show the crystal structure viewed along the $c$ and $b$ axes respectively. In Fig. 9 the unit outlined is half the true projected unit cell, the apparent halving in the $a$ direction being due to a glide plane parallel to (010) with translation $\frac{1}{2} a$. The main feature of the intermolecular packing is the relationship of pairs of molecules about centres of symmetry, so that the whole structure consists of a repetition of dimers. This suggests a rather more powerful force of inter-

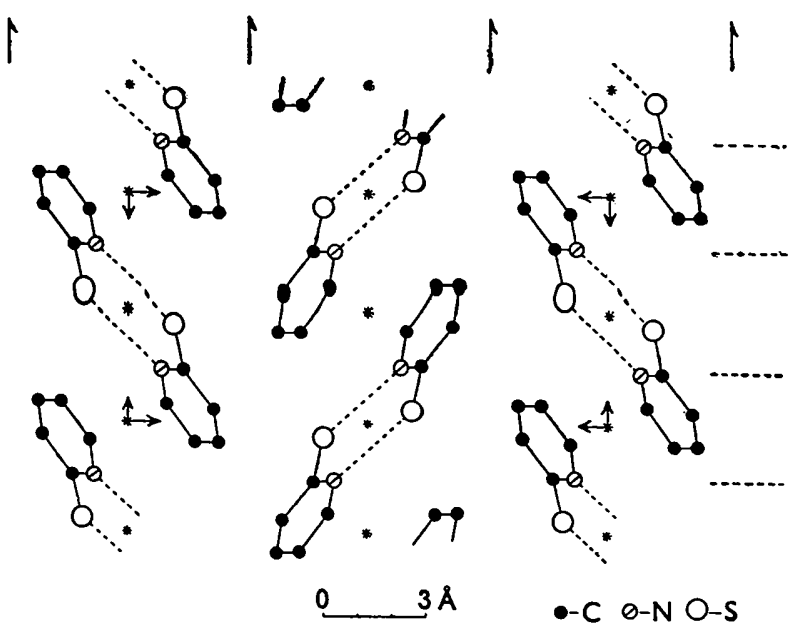

Fig. 8. Structure viewed along $c$ axis. Broken lines represent proposed hydrogen bonds. 
molecular attraction than the van der Waals type and we shall consider the possibility of hydrogen bonding between sulphur and nitrogen atoms.

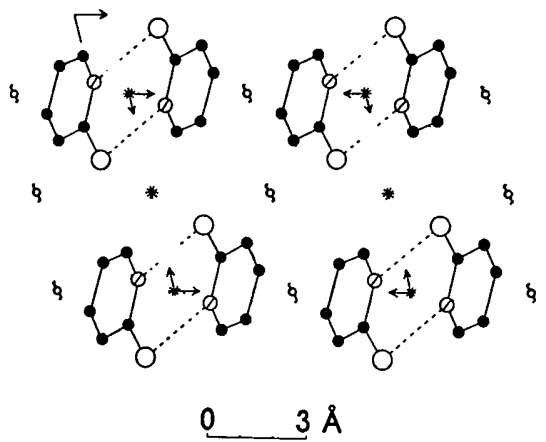

Fig. 9. Structure viewed along $b$ axis with the true unit cell halved in the $a$ direction. Broken lines represent proposed hydrogen bonds. Key as in Fig. 8.

The intermolecular S-N distance is $3 \cdot 26 \AA$, about equal to the sum of the van der Waals radii $(1 \cdot 7+1 \cdot 5)$ $\AA$, so that if a hydrogen bond is to exist between $\mathrm{S}$ and $\mathrm{N}$, a hydrogen atom must be situated very close to the line joining this pair. The magnitudes of the intermolecular angles $\angle \mathrm{C}_{1} \mathrm{NS}^{\prime}$ and $\angle \mathrm{C}_{5} \mathrm{NS}^{\prime}$ (where $\mathrm{S}^{\prime}$ is in the molecule related by a centre of symmetry to that containing $\mathrm{C}_{1}, \mathrm{C}_{5}$ and $\mathrm{N}$ ) are $129^{\circ}$ and $108^{\circ}$ respectively. The sum of these two and $\angle \mathrm{C}_{5} \mathrm{NC}_{1}$ is $359^{\circ}$. Therefore a hydrogen atom covalently bound to $\mathrm{N}$, in order to preserve a planar configuration about this atom, will point almost directly towards $\mathrm{S}^{\prime}$. The distance between this hydrogen atom and $\mathrm{S}^{\prime}$ would then be unlikely to be greater than $(3 \cdot 26-0.8 \AA) \approx 2.5$ $\AA$, compared with the sum of van der Waals radii of about $2.9 \AA$. We may therefore attribute the force of attraction between nitrogen and sulphur atoms to weak hydrogen bonding. Such hydrogen bonds have not been previously reported in crystals and, indeed, their existence is not expected from consideration of the electronegativity of sulphur, which is no greater than that of carbon.*

\section{Comparison with the structure of $\alpha$-pyridone}

The crystal structures of $\alpha$-pyridone and $\alpha$-thiopyridone show some remarkably similar features, beginning with their unit-cell dimensions. The arrangement of

* Strong evidence for the existence of $\mathrm{S}-\mathrm{H}-\mathrm{N}$ bonds in solution has been given by Hopkins \& Hunter (1942) and by Burrows \& Hunter (1952). molecules in the cell is the same, projected on $(001)$. There is, however, a fundamental difference in space in that pyridone molecules are linked in helices by an endless system of hydrogen bonds while thiopyridone molecules are linked only in pairs.

The dimensions of the pyridine rings are closely similar, indicating, as has already been discussed, that the relative weights of contributing resonance structures are the same in both compounds. It may be noted that in both compounds the size of the internal angles of the ring decreases as the average lengths of the bonds forming these angles increases. This effect would appear to be related to the nature of the hybridization of bonding orbitals about each carbon atom, the existence of two bonds which are predominantly single requiring an angle between them which is closer to the tetrahedral than the trigonal angle.

I wish to thank Prof. Sir Lawrence Bragg and Dr W. H. Taylor for facilities provided for this work and for their interest in it. I am greatly indebted to Dr W. Cochran for his encouragement and for much helpful advice. I acknowledge gratefully the award of postgraduate scholarships by the University of New Zealand and the British Council during the tenure of which this work was carried out, and thank the Council of Canterbury University College, New Zealand, for granting three years leave of absence.

\section{References}

Broomhead, J. M. (1948). Acta Cryst. 1, 324.

Burrows, A. A. \& Hunxer, L. (1952). J. Chem. Soc. p. 4118.

Cochran, W. (1948). Acta Cryst. 1, 54.

Cochran, W. (1952). Acta Cryst. 5, 65.

Cochran, W. \& Penfold, B. R. (1952). Acta Cryst. 5, 644.

Cox, E. G. \& Cruickshank, D. W. J. (1948). Acta Cryst. 1, 92 .

Cox, E. G., Gillot, R. J. J. H. \& Jeffrey, G. A. (1949). Acta Cryst. 2, 356.

Evans, M. G. \& Heer, J. DE (1949). Acta Cryst. 2, 363. HARKER, D. \& Kasper, J. S. (1948). Acta Cryst. 1, 70. Hopkins, G. \& Hunter, L. (1942). J. Chem. Soc. p. 638. Hughes, E. W. (1941). J. Amer. Chem. Soc. 63, 1737.

James, R. W. \& Brindley, G. W. (1931). Phil. Mag. (7), 12,81 .

Kasper, J. S., Lucht, C. M. \& HaRkER, D. (1950). Acta Cryst. 3, 439.

Penfold, B. R. (1953). Acta Cryst. 6, 591.

Skinner, H. A. (1945). Trans. Faraday Soc. 41, 645.

Wruson, A. J. C. (1942). Nature, Lond. 150, 151. 